\title{
Crescimento e qualidade de mudas de cedro (Cedrela fissilis L.) em função do substrato e sombreamento
}

Growth and seedlings quality of Cedrela fissilis L. under different substrates and shading

\author{
A. D. V. Gomes*; A. L. O. Freire \\ Universidade Federal de Campina Grande, Centro de Saúde e Tecnologia Rural, Unidade Acadêmica de Engenharia \\ Florestal, 58700-970, Campus de Patos - PB, Brasil \\ arturvieiral@hotmail.com
}

(Recebido em 15 de outubro de 2019; aceito em 30 de novembro de 2019)

\begin{abstract}
Este trabalho teve como objetivo avaliar o crescimento inicial e a qualidade de mudas de cedro em função de diferentes substratos e de níveis de sombreamento. $\mathrm{O}$ experimento foi distribuído em delineamento inteiramente casualizado, esquema fatorial 3 x 5, com três níveis sombreamento e cinco substratos, com cinco repetições e quatro plantas por unidade amostral. As plantas foram mantidas a $0 \%$ (sol pleno), 50\% e 70\% de sombreamento e os substratos utilizados foram uma mistura de solo (S), pó de coco (PC), casca de arroz carbonizada (CA), rejeito de vermiculita (RV) e esterco bovino (EB) em proporções variadas, formando quatro combinações de substratos [SEB (S70\% + EB30\%); SCAEB (S70\% + CA15\% + EB 15\%); SPCEB $(\mathrm{S} 70 \%+\mathrm{PC} 15 \%+\mathrm{EB} 15 \%)$ e SRVEB $(\mathrm{S} 70 \%+\mathrm{RV} 15 \%+\mathrm{EB} 15 \%)]$, mais um substrato comercial Plantmax ${ }^{\circledR}$ (PLX). O sombreamento 70\%, independente do substrato empregado, proporcionou os maiores valores de altura de plantas, diâmetro do coleto, área foliar e massa seca das mudas. O cultivo a sol pleno influenciou negativamente o crescimento e a qualidade das mudas, os quais foram favorecidos pelo nível de $50 \%$ de luminosidade, nos substratos SCAEB, SPCEB, SEB e PLX, por apresentar maior valor de IQD. A utilização de casca de arroz carbonizada, pó de coco e rejeito de vermiculita tem potencial para serem usados na composição de substratos para produção de mudas de C. fissilis L., em adição ao solo e esterco bovino.

Palavras-chave: viveiros florestais, produção de mudas, luminosidade.
\end{abstract}

This study aimed to evaluate the initial growth and quality of Cedrela fissilis seedlings under different substrates and shading. The experiment was distributed in a completely randomized design, in $3 \times 5$ factorial scheme, with three levels of shading (70\% 50\% and 0\%) and five substrates, with five replications and four plants per sample unit. The substrates were formed from soil (S), coconut powder (CP), carbonized rice husk $(\mathrm{RH})$, vermiculite tailings (VT) and bovine manure (BM) in the different proportions [SBM (S70\%+ BM30\%); SRHEB (S70\% + RH15\% + BM 15\%); SCPBM (S70\% + CP15\% + BM15\%) e SVTBM (S70\% + VT15\% + BM15\%)], and Plantmax ${ }^{\circledR}$ (PLX), a commercial substrate. Shading 70\%, regardless of substrate, provided the highest values for plant height, stem diameter, leaf area and seedling dry mass. The $0 \%$ shading level had a negative influence on the growth and quality of the seedlings, which were favored by the $50 \%$ level of shading in the SRHBM substrates, SCPBM, because it had a higher IQD value. The use of carbonized rice hulls, coconut powder and vermiculite tailings has the potential to be used in thecomposition of substrates for seedling production of $C$. fissilis $L$.

Keywords: forest nursery, seedling production, light.

\section{INTRODUÇÃO}

O cedro (Cedrela fissilis L.), popularmente conhecido como cedro-vermelho ou cedro rosa, tem ocorrência principalmente na Amazônia, Cerrado e Mata Atlântica e é utilizado para os mais diversos fins madeireiros e não madeireiros e, devido à exploração indiscriminada, encontra-se em vias de extinção [1,2].

No processo de produção de mudas, diversos fatores podem influenciar o crescimento e a qualidade das mesmas, dentre os quais se destacam o sombreamento e o substrato [3]. As condições ambientais em que as plantas se desenvolvem interferem diretamente no seu crescimento, de acordo com suas habilidades de se adaptarem às diferentes intensidades de radiação, podendo expressar um entre os dois mecanismos de respostas sob restrição de luz: tolerância e escape [4]. As espécies consideradas tolerantes ao sombreamento são capazes de sobreviver por longos períodos sob o dossel, por conseguirem desenvolver adaptações para a realização da fotossíntese, enquanto que as 
plantas que demostram mecanismo de escape tendem a maximizar a interceptação de luz adaptando seu crescimento e ocupando lacunas do dossel [5]. Em espécies arbóreas nativas, as informações referentes à intensidade luminosa durante a fase de viveiro são incipientes devido ao fato de que pode haver mudanças significativas no fotoperíodo entre as regiões de estudo, possibilitando alterações significativas das respostas de desenvolvimento para uma mesma espécie estudada [6].

$\mathrm{O}$ uso de telados que permitem o controle da radiação solar tem auxiliado nos estudos de crescimento de plantas que objetivam entender o comportamento ecofisiológico de espécies submetidas a diferentes condições de luminosidade, indicando, assim, as melhores condições de luminosidade para o cultivo de determinada espécie. Melhores índices de qualidade em mudas de Caesalpinia echinata Lam, quando submetidas a maiores níveis sombreamento [7], enquanto que em mudas Sclerolobium paniculatum Vogel o melhor crescimento ocorreu quando as plantas foram submetidas à maior incidência de luz [8]. Percebe-se, então, que as necessidades de luminosidade variam de espécie para espécie.

Outro fator que deve obrigatoriamente ser levado em consideração na produção de mudas em viveiro é o substrato [9]. Este deve proporcionar condições adequadas à germinação e ao crescimento inicial das mudas, apresentando boas características químicas, densidade, capacidade de retenção de água, aeração e drenagem adequadas, de modo a evitar acúmulo de umidade, além de estar isento de patógenos, plantas daninhas e de substâncias tóxicas [3].

No empreendimento florestal há a necessidade de utilização de substratos que reduzam os custos e forneçam condições necessárias para um bom desempenho da muda. Nesse contexto, vários são os estudos no sentido de procurar produtos que possam ser utilizados na composição de substrato, tais como lodo de esgoto, casca de arroz carbonizada, palha de café in natura, esterco bovino, vermiculita, fibra de coco, areia, entre outros $[9,10,11]$.

Em virtude da carência de informações na literatura especializada acerca do substrato ideal para a produção de mudas, assim como o comportamento de mudas de cedro sob sombreamento, desenvolveu-se a presente pesquisa com o objetivo de avaliar a influência dos diferentes tipos de substrato e níveis de sombreamento no crescimento inicial e na qualidade de mudas de cedro.

\section{MATERIAL E MÉTODOS}

A pesquisa foi conduzida no Viveiro Florestal da Unidade Acadêmica de Engenharia Florestal, do Centro de Saúde e Tecnologia Rural da Universidade Federal de Campina Grande (CSTR/UFCG), Campus de Patos, coordenadas geográficas 703"35” S e 37¹6"29" O. O clima da região é do tipo Bsh, segundo Köppen, classificado como semiárido quente, precipitação média anual de $750 \mathrm{~mm}$, umidade relativa do ar em torno de $80 \%$ e temperatura média anual é de $25,5^{\circ} \mathrm{C}$ [12].

O delineamento experimental adotado foi inteiramente cazualizado (DIC), com os tratamentos distribuídos em esquema fatorial 3 x 5 , sendo três níveis de sombreamento e cinco substratos, com cinco repetições e quatro plantas por unidade amostral, totalizando 300 plantas. Os níveis de sombreamento foram $0 \%$ (sol pleno), $50 \%$ e $70 \%$, obtidos com o auxílio de telas tipo sombrite.

Os substratos utilizados foram compostos de uma mistura (v/v) de terra de sub-solo (S), pó de fibra de coco (PC), casca de arroz carbonizada (CA), rejeito de vermiculita (RV) e esterco bovino curtido (EB), em quatro combinações, mais um substrato comercial Plantmax ${ }^{\circledR}$, a saber: PLX: Plantmax ${ }^{\circledR}$; SEB (S70\% + EB30\%); SCAEB (S70\% + CA15\% + EB 15\%); SPCEB (S70\% + $\mathrm{PC} 15 \%+\mathrm{EB} 15 \%)$ e SRVEB (S70\% + RV15\% + EB15\%).

$\mathrm{O}$ rejeito de vermiculita foi obtido na Mineradora Pedra Lavrada, localizada no município de Santa Luzia, PB. A palha de arroz foi adquirida em fábrica beneficiadora de alimentos, localizada na cidade de Patos-PB, e posteriormente carbonizada. O pó de coco foi adquirido na Universidade Federal do Vale do São Francisco (UNIVASF), Petrolina-PE e, antes da sua utilização, foi lavado com a finalidade de retirada do excesso de sais $\left(\mathrm{Na}^{+}\right)$e posteriormente submetido à secagem à sombra.

Após a preparação, amostras dos substratos foram submetidas à análise química, cujos resultados encontram-se na Tabela 1 . 
Tabela 1: Análise química dos substratos utilizados no experimento com Cedrela fissilis.

\begin{tabular}{lllllllllll}
\hline \multirow{2}{*}{ Substrato } & $\mathrm{pH}$ & $\mathrm{M} . \mathrm{O}$. & $\mathrm{P}$ & $\mathrm{K}$ & $\mathrm{Na}$ & $\mathrm{Ca}$ & $\mathrm{Mg}$ & $\mathrm{H}+\mathrm{Al}$ & $\mathrm{T}$ & $\mathrm{V}$ \\
\cline { 2 - 10 } & $\mathrm{H}_{2} \mathrm{O}$ & $\mathrm{dag} / \mathrm{kg}$ & $-------\mathrm{mg} / \mathrm{dm}^{-3}------$ & $--------\mathrm{cmol}_{\mathrm{c}}$ & $\mathrm{dm}^{-3}--------$ & $\%$ \\
\hline PLX & 5,7 & 12,8 & 215 & 450 & 260 & 13,8 & 8,7 & 4,7 & 29,5 & 84,1 \\
SEB & 6,8 & 2,5 & 150 & 340 & 290 & 10,1 & 2,7 & 1,5 & 14,9 & 90,9 \\
SCAEB & 6,5 & 2,4 & 215 & 280 & 160 & 8,0 & 1,9 & 1,5 & 11,3 & 88,3 \\
SPCEB & 6,5 & 1,4 & 187 & 210 & 130 & 6,0 & 2,0 & 1,6 & 9,1 & 85,1 \\
SRVEB & 7,0 & 1,7 & 177 & 260 & 450 & 10,4 & 3,9 & 1,3 & 16,9 & 92,9 \\
\hline
\end{tabular}

M.O: material orgânica; P: fósforo; K: potássio; Na: sódio; Ca: cálcio; Mg: magnésio; H: hidrogênio; Al: alumínio; T: capacidade de troca catiônica efetiva; V: saturação por bases; PLX: Plantmax®; SEB: S70\% + EB30\%; SCAEB: S70\% + CA15\% + EB 15\%; SPCEB: S70\% + PC15\% + EB15\% e SRVEB: S70\% + $\mathrm{RV} 15 \%+\mathrm{EB} 15 \%$.

Os substratos foram colocados em vasos, os quais foram confeccionados em garrafas PET, com capacidades para 1,5 L, pintadas externamente com tinta preta com a finalidade de evitar $\mathrm{o}$ desenvolvimento de algas nos substratos. As sementes foram previamente colocadas para germinar em caixas gerbox forradas com duas folhas de papel germitest, umedecidas com $10 \mathrm{~mL}$ de água destilada, e posteriormente transferidas para câmara de germinação com temperatura de $25{ }^{\circ} \mathrm{C}$. Após a emissão da radícula, as sementes foram transferidas para os recipientes contendo os substratos.

O experimento foi encerrado aos 180 dias após a emergência, momento em que foram determinadas a altura das plantas (AP) e o diâmetro do caule (DC), medido a 1,0 cm do nível do solo. Em seguida, as folhas foram colhidas e levadas para digitalização em scanner de mesa, sendo os dados utilizados para determinação da área foliar (AF) utilizando-se o software DDA (Determinador Digital de Áreas) [13]. Posteriormente, as folhas, o caule e as raízes foram colocados em sacos de papel para secagem em estufa de circulação de ar forçada a $65^{\circ} \mathrm{C}$ e, após atingirem a massa constante, foram submetidos à pesagem para a determinação da massa seca da parte aérea (MSPA), das raízes (MSR) e total (MST).

O índice de qualidade de Dickson (IQD) foi calculado através da fórmula proposta por Dickson et al. (1960) [14]:

$$
\left(\mathrm{IQD}=\frac{\mathrm{MST}(\mathrm{g})}{\left[\frac{\mathrm{AP}(\mathrm{cm})}{\mathrm{DC}(\mathrm{mm})}\right]+\left[\frac{\mathrm{MSPA}(\mathrm{g})}{\mathrm{MSR}(\mathrm{g})}\right]}\right)
$$

Os dados foram submetidos à análise de variância usando-se o Software ASSISTAT [15], e as médias comparadas pelo teste de Tukey a $5 \%$ de probabilidade.

\section{RESULTADOS E DISCUSSÃO}

Foi verificado efeito significativo do sombreamento e interação significativa dos tratamentos na altura das plantas (Tabela 2), sendo que os maiores valores foram constatados nas plantas submetidas a $70 \%$ de sombreamento, com redução à medida que o nível de luz incidente sobre as plantas aumentava. 
Tabela 2: Altura de plantas de cedro em função do substrato e níveis de sombreamento.

\begin{tabular}{lcccc}
\hline \multicolumn{4}{c}{ ALTURA DE PLANTAS (cm) } \\
\cline { 1 - 4 } SUBSTRATO & \multicolumn{3}{c}{ SOMBREAMENTO $(\mathbf{\%})$} & \multirow{2}{*}{ MÉDIA } \\
\cline { 2 - 4 } & $\mathbf{0}$ & $\mathbf{5 0}$ & $\mathbf{7 0}$ & \\
\hline PLX & $13,55 \mathrm{bA}$ & $11,95 \mathrm{bA}$ & $30,75 \mathrm{aAB}$ & $\mathbf{1 8 , 7 5}$ \\
SEB & $11,60 \mathrm{bA}$ & $15,60 \mathrm{bA}$ & $36,50 \mathrm{aA}$ & $\mathbf{2 1 , 2 3}$ \\
SCAEB & $11,75 \mathrm{bA}$ & $15,30 \mathrm{bA}$ & $27,80 \mathrm{aB}$ & $\mathbf{1 8 , 2 8}$ \\
SPCEB & $11,40 \mathrm{bA}$ & $13,00 \mathrm{bA}$ & $31,00 \mathrm{aAB}$ & $\mathbf{1 8 , 4 6}$ \\
SRVEB & $11,42 \mathrm{bA}$ & $14,20 \mathrm{bA}$ & $35,33 \mathrm{aAB}$ & $\mathbf{2 0 , 3 2}$ \\
\hline MÉDIA & $\mathbf{1 1 , 8 8}$ & $\mathbf{1 2 , 9 3}$ & $\mathbf{2 5 , 0 0}$ & \\
\hline
\end{tabular}

PLX: Plantmax ${ }^{\circledR}$; SEB: S70\% + EB30\%; SCAEB: S70\% + CA15\% + EB 15\%; SPCEB: S70\% + PC15\% + EB15\% e SRVEB: S70\% + RV15\% + EB15\%. Médias seguidas de mesma letra, maiúscula na coluna e minúscula na linha, não diferem entre si pelo teste de Tukey $(\mathrm{p} \leq 0,05)$.

As plantas no substrato SEB e a $70 \%$ de sombreamento foram mais altas $(36,5 \mathrm{~cm})$, ao passo que aquelas mantidas a sol pleno e crescendo no substrato SPCEB, foram as menores $(11,40 \mathrm{~cm})$, correspondendo a uma redução de $69 \%$ na altura das plantas. Salienta-se que o substrato SEB apresentava maior porcentagem de esterco bovino em relação ao SCAEB, o qual contribuiu para teores mais elevados de $\mathrm{K}, \mathrm{Ca}$ e $\mathrm{Mg}$, concorrendo assim para maior crescimento das plantas, evidenciando que baixos teores de nutrientes no meio reduzem o crescimento das plantas [16].

Quanto ao diâmetro do caule (Tabela 3), verificou-se ampla variação nos valores, semelhante aos obtidos na altura das plantas. Os substratos SRVEB e SCAEB se destacaram frente aos demais, enquanto que, a exemplo do verificado na altura das plantas, os tratamentos $70 \%$ e $25 \%$ de sombreamento proporcionaram, respectivamente, plantas com maior e menor diâmetro do caule, respectivamente.

Tabela 3: Diâmetro do caule de cedro em função do substrato e níveis de sombreamento.

\begin{tabular}{lcccc}
\hline \multicolumn{4}{c}{ DIÂMETRO DO CAULE (mm) } \\
\cline { 1 - 4 } SUBSTRATO & \multicolumn{3}{c}{ SOMBREAMENTO (\%) } & \multirow{2}{*}{ MÉDIA } \\
\cline { 2 - 4 } & $\mathbf{0}$ & $\mathbf{5 0}$ & $\mathbf{7 0}$ & \\
\hline PLX & $8,85 \mathrm{bA}$ & $8,43 \mathrm{bC}$ & $9,60 \mathrm{aD}$ & $\mathbf{8 , 9 6}$ \\
SEB & $7,22 \mathrm{bB}$ & $9,27 \mathrm{bAB}$ & $10,66 \mathrm{aBC}$ & $\mathbf{9 , 0 5}$ \\
SCAEB & $7,32 \mathrm{cB}$ & $9,74 \mathrm{bA}$ & $11,22 \mathrm{aB}$ & $\mathbf{9 , 4 3}$ \\
SPCEB & $7,78 \mathrm{cB}$ & $9,06 \mathrm{bABC}$ & $10,12 \mathrm{aCD}$ & $\mathbf{8 , 9 9}$ \\
SRVEB & $7,46 \mathrm{cB}$ & $8,49 \mathrm{bC}$ & $12,14 \mathrm{aA}$ & $\mathbf{9 , 3 6}$ \\
\hline MÉDIA & $\mathbf{6 , 8 9}$ & $\mathbf{7 , 7 2}$ & $\mathbf{9 , 2 2}$ & \\
\hline
\end{tabular}

PLX: Plantmax ${ }^{\circledR}$; SEB: S70\% + EB30\%; SCAEB: S70\% + CA15\% + EB 15\%; SPCEB: S70\% + PC15\% + EB15\% e SRVEB: S70\% + RV15\% + EB15\%. Médias seguidas de mesma letra, maiúscula na coluna e minúscula na linha, não diferem entre si pelo teste de Tukey $(\mathrm{p} \leq 0,05)$.

Avaliando diferentes substratos e sombreamentos em mudas de Tocoyena formosa, foi observado que o maior crescimento da parte aérea poderia estar relacionado com a maior quantidade de nutrientes no substrato e/ou em função da matéria orgânica, por promover maior retenção e disponibilidade de água para manter a turgescência e metabolismo da parte aérea [17]. Nessa mesma linha de pensamento, em Carica papaya L. constatou-se que menor teor de matéria orgânica presente no substrato interferiu negativamente no crescimento em altura das plantas [18]. Resultados semelhantes foram observados na produção de mudas de Schizolobium amazonicum Huber ex Ducke, com maior incremento na altura das plantas e no diâmetro do caule [19]. Em mudas de aroeira (Myracrondruon urundeuva Allemão), os substratos que apresentavam esterco bovino na sua composição proporcionaram a obtenção dos maiores valores de altura das plantas, diâmetro do coleto e acúmulo de massa seca [20]. Contrariamente, a ausência de matéria orgânica não interferiu no crescimento de mudas de gabiroba (Campomanesia adantium (Cambess.) $\mathrm{O}$. 
Berg.), uma vez que os substratos latossolo vermelho+areia e latossolo vermelho+Bioplant ${ }^{\circledR}$ proporcionaram melhor crescimento das mudas [21].

O cedro é uma planta classificada como ombrófila na sua fase inicial [22], o que pode explicar uma tendência a um maior crescimento nos estágios iniciais sob condições de baixa luminosidade, o que foi verificado entre as plantas submetidas a $70 \%$ de sombreamento que apresentaram os maiores valores de altura e diâmetro do caule. Plantas submetidas a uma baixa luminosidade tendem a investir normalmente de forma mais intensa em altura [23]. Esse evento pode ser explicado principalmente devido ao fenômeno de estiolamento e/ou aos mecanismos adaptativos entre diversas espécies de plantas que promovem maior crescimento em ambientes com restrição de luz $[23,24]$.

Outro fator a ser considerado é que o ambiente telado proporciona condições mais amenas, formando um microclima ao elevar a umidade relativa do ar e menor temperatura, quando comparadas às condições externas, dando condições para um maior crescimento em uma região com condições de temperaturas elevadas e baixos níveis de umidade relativa do ar, como o semiárido brasileiro. Temperaturas mais amenas em decorrência do sombreamento favorecem a abertura dos estômatos, aumentando as trocas gasosas e, consequentemente, a fixação de carbono pelas plantas [25].

Aumento no nível de luminosidade proporcionou acréscimo na área foliar das plantas, destacando-se o tratamento 70\% de sombra, associado aos substratos SEB e SRVEB (Tabela 4). Vale ressaltar a associação desse último substrato e 50\% de luz, a qual proporcionou o a obtenção do terceiro valor de área foliar. $\mathrm{O}$ valor mais baixo de área foliar foi obtido nas plantas a pleno sol, no substrato SPCEB.

Tabela 4: Área foliar total de plantas de cedro em função do substrato e níveis de sombreamento.

\begin{tabular}{lcccc}
\hline \multirow{4}{*}{ SUBSTRATO } & \multicolumn{3}{c}{ ÁREA FOLIAR $\left(\mathbf{c m}^{2}\right)$} & \multirow{2}{*}{ MÉDIA } \\
\cline { 2 - 4 } & $\mathbf{0}$ & $\mathbf{5 0}$ & $\mathbf{7 0}$ & \\
\hline PLX & $101,39 \mathrm{bA}$ & $96,43 \mathrm{bCDE}$ & $358,03 \mathrm{aB}$ & $\mathbf{1 8 5 , 2 8}$ \\
SEB & $64,89 \mathrm{cA}$ & $213,34 \mathrm{bBCD}$ & $566,28 \mathrm{aA}$ & $\mathbf{2 8 1 , 5 0}$ \\
SCAEB & $88,27 \mathrm{bA}$ & $357,76 \mathrm{aAB}$ & $287,87 \mathrm{aBC}$ & $\mathbf{2 4 4 , 6 3}$ \\
SPCEB & $39,58 \mathrm{bA}$ & $237,12 \mathrm{aABC}$ & $346,93 \mathrm{aBC}$ & $\mathbf{2 0 7 , 8 8}$ \\
SRVEB & $81,14 \mathrm{cA}$ & $405,33 \mathrm{bA}$ & $554,48 \mathrm{aA}$ & $\mathbf{3 4 6 , 9 8}$ \\
\hline MÉDIA & $\mathbf{5 9 , 2 3}$ & $\mathbf{1 6 8 , 3 4}$ & $\mathbf{2 9 1 , 3 1}$ & \\
\hline
\end{tabular}

PLX: Plantmax ${ }^{\circledR}$; SEB: S70\% + EB30\%; SCAEB: S70\% + CA15\% + EB 15\%; SPCEB: S70\% + PC15\% + EB15\% e SRVEB: S70\% + RV15\% + EB15\%. Médias seguidas de mesma letra, maiúscula na coluna e minúscula na linha, não diferem entre si pelo teste de Tukey $(\mathrm{p} \leq 0,05)$.

Resultados semelhantes foram verificados em mudas de Pterogyne nitens Tull. conduzidas sob diferentes níveis de luminosidade [10], em que ocorreu acréscimo de $47 \%$ na área foliar em plantas submetidas a 70\% de luminosidade quando comparados às plantas a pleno sol. Esse comportamento também foi constatado em plantas de Eucalyptus dunnii, sob sombreamento, promovendo a obtenção de aproximadamente $12 \%$ a mais em área foliar quando comparado às plantas que não estavam sob restrição de luminosidade [26].

A área foliar é uma das variáveis morfológicas mais importantes na análise de crescimento de uma muda, podendo ser considerada um índice de produtividade, dada à importância dos órgãos fotossintetizantes na produção biológica [27]. Espera-se que espécies de plantas tolerantes ao sombreamento compensem a baixa disponibilidade de luz aumentando sua área foliar e superfície fotossintetizante, elevando assim as taxas de produção de fotoassimilados, o que lhes assegura um rendimento fotossintético mais eficiente em baixas intensidades de luz [28]. Essa é uma característica esperada por espécies vegetais de estágios sucessionais mais avançados como as secundárias tardias e clímax que se adaptam melhor em ambientes de menor intensidade luminosa [2]. Esse é o comportamento esperado pelo cedro, objeto deste estudo, uma espécie classificada entre secundária a clímax [22, 24]. 
Em relação à massa seca das plantas, aumento no nível de sombreamento promoveu elevação nas massas secas da parte aérea e das raízes e, consequentemente, na massa seca total das plantas, independente do substrato (Tabela 5).

Tabela 5. Massa seca de plantas de cedro em função do substrato e níveis de sombreamento.

\begin{tabular}{|c|c|c|c|c|}
\hline \multicolumn{5}{|c|}{ MASSA SECA DA PARTE AÉREA (g) } \\
\hline \multirow{2}{*}{ SUBSTRATOS } & \multicolumn{3}{|c|}{ SOMBREAMENTO $(\%)$} & \multirow{2}{*}{ MÉDIA } \\
\hline & $\mathbf{0}$ & 50 & 70 & \\
\hline PLX & $4,27 \mathrm{bA}$ & $4,64 \mathrm{bCD}$ & 8,85 aABC & 5,92 \\
\hline SEB & $2,73 \mathrm{cA}$ & $6,44 \mathrm{aAB}$ & $11,55 \mathrm{aAB}$ & 6,91 \\
\hline SCAEB & $3,29 \mathrm{bA}$ & $8,80 \mathrm{aAB}$ & $8,43 \mathrm{aABC}$ & 6,86 \\
\hline SPCEB & $1,90 \mathrm{bA}$ & $7,40 \mathrm{aBC}$ & 7,69 aABC & 5,66 \\
\hline SRVEB & $2,98 \mathrm{bA}$ & $6,62 \mathrm{aAB}$ & $12,04 \mathrm{aA}$ & 7,21 \\
\hline MÉDIA & 2,45 & 4,74 & 7,22 & \\
\hline \multicolumn{5}{|c|}{ MASSA SECA DE RAÍZES (g) } \\
\hline \multirow{2}{*}{ SUBSTRATO } & \multicolumn{3}{|c|}{ SOMBREAMENTO (\%) } & \multirow{2}{*}{ MÉDIA } \\
\hline & $\mathbf{0}$ & 50 & 70 & \\
\hline PLX & $2,32 \mathrm{cA}$ & $4,12 \mathrm{aA}$ & $3,80 \mathrm{bBC}$ & 3,75 \\
\hline SEB & $1,69 \mathrm{bAB}$ & $4,19 \mathrm{aA}$ & $5,53 \mathrm{aA}$ & $\mathbf{3 , 5 0}$ \\
\hline SCAEB & $1,93 \mathrm{cAB}$ & $4,64 \mathrm{aA}$ & $4,28 \mathrm{bBC}$ & 3,95 \\
\hline SPCEB & $1,52 \mathrm{cABC}$ & $4,19 \mathrm{aA}$ & $3,21 \mathrm{bC}$ & 2,97 \\
\hline SRVEB & $1,68 \mathrm{bAB}$ & $4,94 \mathrm{aA}$ & $4,58 \mathrm{aAB}$ & 3,73 \\
\hline MÉDIA & 1,48 & 3,11 & 3,23 & \\
\hline \multicolumn{5}{|c|}{ MASSA SECA TOTAL (g) } \\
\hline \multirow{2}{*}{ SUBSTRATO } & \multicolumn{3}{|c|}{ SOMBREAMENTO (\%) } & \multirow{2}{*}{ MÉDIA } \\
\hline & $\mathbf{0}$ & 50 & 70 & \\
\hline PLX & $6,59 \mathrm{bA}$ & $8,76 \mathrm{bC}$ & $12,65 \mathrm{aAB}$ & 9,33 \\
\hline SEB & $4,42 \mathrm{cA}$ & $10,63 \mathrm{bBC}$ & $17,08 \mathrm{aA}$ & 10,71 \\
\hline SCAEB & $5,22 \mathrm{bA}$ & $13,44 \mathrm{aAB}$ & $12,71 \mathrm{aAB}$ & 10,49 \\
\hline SPCEB & $3,42 \mathrm{bA}$ & $11,59 \mathrm{aBC}$ & $10,91 \mathrm{aAB}$ & 8,64 \\
\hline SRVEB & $4,66 \mathrm{cA}$ & $11,56 \mathrm{bA}$ & $16,62 \mathrm{aA}$ & 10,95 \\
\hline MÉDIA & 4,02 & 7,82 & 10,30 & \\
\hline
\end{tabular}

PLX: Plantmax ${ }^{\circledR}$; SEB: S70\% + EB30\%; SCAEB: S70\% + CA15\% + EB 15\%; SPCEB: S70\% + PC15\% + EB15\% e SRVEB: S70\% + RV15\% + EB15\%. Médias seguidas de mesma letra, maiúscula na coluna e minúscula na linha, não diferem entre si pelo teste de Tukey $(\mathrm{p} \leq 0,05)$.

Os tratamentos que proporcionaram maiores valores de massa seca das plantas foram SEB e SRVEB, a 70\% de sombreamento e, semelhante ao verificado nos parâmetros morfológicos anteriores, as plantas mantidas a pleno sol apresentaram os valores mais baixos.

Comportamentos que corroboram os obtidos neste estudo foram verificados em plantas de Hymenaea courbaril e Enterolobium contortisiliquum [6] e em Pterogyne nitens Tull. [7], os quais constataram decréscimo na produção de massa seca em plantas cultivadas em pleno sol. Altas irradiâncias e a exposição prolongada podem ser prejudiciais às plântulas ou mesmo provocar sua morte, devido a uma provável fotoinibição, uma vez que estas podem absorver mais fótons de luz do que conseguem assimilar [29].

Ao se analisar a razão peso da massa seca da raiz/peso da massa seca da parte aérea (Tabela 6) (MSR/MSPA), verifica-se que as plantas submetidas a 50\% de sombreamento se destacaram em relação aos demais níveis. Além disso, as plantas mantidas nos substratos PLX e SRVEB, a 50\% de sombra, apresentaram os maiores valores da razão MSR/MSPA, refletindo o menor crescimento da parte aérea em detrimento das raízes. 
devido ao maior crescimento e melhor relação do sistema radicular e aéreo, assim como para o IQD, sob condições de maior luminosidade [7].

\section{CONCLUSÃO}

A manutenção das plantas de cedro a sol pleno interfere negativamente no seu crescimento inicial.

O sombreamento a $70 \%$ proporcionou a obtenção de plantas mais altas, com maiores diâmetros do caule e massa seca.

A casca de arroz carbonizada, o pó de coco e o rejeito de vermiculita têm potencial para serem usados na composição de substratos para produção de mudas de cedro, em adição ao solo e esterco bovino.

A qualidade das mudas de cedro é favorecida pelo cultivo em $50 \%$ de sombreamento, nos substratos SCAEB e SPCEB, por apresentar maior valor de IQD, podendo garantir maiores chances de sobrevivência ao serem transplantadas para o campo.

\section{AGRADECIMENTO}

Os autores agradecem à Coordenação de Aperfeiçoamento de Pessoal de Nível Superior - CAPES pela bolsa de estudos ao primeiro autor.

\section{REFERÊNCIAS BIBLIOGRÁFICAS}

1. Almeida AN, Angelo H, Silva JGL, Hoeflich VA Mercado de madeiras tropicais: substituição na demanda de exportação Acta Amaz. 2010 Mar;40(1)119-126, doi: 10.1590/S0044-59672010000100015.

2. Carvalho PER. Espécies arbóreas brasileiras. Brasília, DF: Embrapa informação tecnológica: colombo, PR: Embrapa Floresta, p. 211-215, 2008.

3. Dantas BF, Lopes AP, Silva FFS, Lúcio AA, Batista PF, Pires MMML, Aragão CA. Taxas de crescimento de mudas de catingueira submetidas a diferentes substratos e sombreamentos. Rev Arv. 2009 Mai;33(3)413-423, doi: 10.1590/S0100-6762009000300003.

4. Ruberti I, Sessa G, Ciolfi A, Possenti M, Carabelli M, Morelli G. Plant adaptation to dynamically changing environment: the shade avoidance response. Biotech Adv. 2012 Out;30(5)1047-1058, doi: 10.1016/j.biotechadv.2011.08.014.

5. César FRCF, Matsumoto SN, Viana AES, Bonfim JA. Crescimento inicial e qualidade de mudas de Pterogyne nitens Tull. conduzidas sob diferentes níveis de restrição luminosa artificial. Ci Fl. 2014 Abr;24(2)357-366, doi: 10.5902/1980509814573.

6. Lima ALS, Zanella' F, Castro LDM. Crescimento de Hymenaea courbaril L. var. stilbocarpa (Hayne) Lee et Lang. e Enterolobium contortisiliquum (Vell.) Morong (Leguminosae) sob diferentes níveis de sombreamento. Acta Amaz. 2010 Mar;40(1)43-48, doi: 10.1590/S0044-59672010000100006.

7. Aguiar FFA, Kanashiro S, Tavares AR, Nascimento TDR, Rocco FM. Crescimento de mudas de paubrasil (Caesalpinia echinata Lam.), submetidas a cinco níveis de sombreamento. Rev Ceres. 2011 Nov;58(6):729-734, doi: 10.1590/S0034-737X2011000600008.

8. Freitas GA, Melo AV, Pereira MAB, Andrade CAO, Lucena GN, Silva RR. Influência do sombreamento na qualidade de mudas de Sclerolobium paniculatum Vogel para recuperação de área degradada. J Biotechnol Biodiv. 2012 Ago;3(3)5-12.

9. Caldeira MVW, Delarmelina WM, Faria JCT, Juvanhol RS. Substratos alternativos na produção de mudas de Chamaecrista desvauxii L. Rev Arv. 2013 Jan;37(1)31-39, doi: 10.1590/S0100-67622013000100004.

10. Costa E, Dias JG, Lopes KG, Binotti FFS, Cardoso ED. Telas de sombreamento e substratos na produção de mudas de Dipteryx alata Vog. Flor e Amb. 2015 Jul;22(3)416-425, doi: 10.1590/2179-8087.071714.

11. Dutra TR, Grazziotti PH, Santana RC, Massad MD. Qualidade de mudas de copaíba produzidas em diferentes substratos e níveis de sombreamento. Floresta. 2015 Mar;45(3)635-644, doi: 10.5380/rf.v45i3.35686.

12. Monteiro DR, Oliveira DGH, Alencar ZEV, Farias SAR. Levantamento pluviométrico do município de Patos-PB nos últimos 16 anos. I Workshop Internacional Sobre Água no Semiárido Brasileiro. Vol. 1. 2015.

13. Ferreira OGL, Rossi FD, Andrighetto C. DDA: Determinador Digital de Áreas - Software para determinação de área foliar, índice de área foliar e área de olho de lombo. Versão 1.2. Santo Augusto: IFFarroupilha, 2008. 
14. Dickson A, Leaf AL, Hosner JF. Quality appraisal of white spruce and white pine seedling stock in nurseries. The For Chron. 1960 Jan;36(1)10-13, doi: 10.5558/tfc36010-1.

15. Silva FAS, Azevedo CAV. Versão do programa computacional Assistat para o sistema operacional Windows. Rev Bras Prod Agroind. 2002 Mar;4(1)71-78.

16. Lavres-Júnior J, Boaretto RM, Silva MLS, Correia D, Cabral PC, Malavolta E. Deficiências de macronutrientes no estado nutricional da mamoeira cultivar Iris. Pesq Agrop Bras. 2005 Fev;40(2)145151, doi: 10.1590/S0100-204X2005000200007

17. Bonamigo T, Scalon SPQ, Pereira ZV. Substratos e níveis de luminosidade no crescimento inicial de mudas de Tocoyena formosa (Cham. \& Schltdl.) K. Schum. (RUBIACEAE). Ci Fl. 2016 Abr;26(2)501511, doi.org/10.5902/1980509822750

18. Araújo EBG, Almeida LLS, Fernandes PF, Sá FVS, Nobre RG, Paiva EP, Mesquita EF, Portela JC. Fontes e doses de matéria orgânica na produção de mudas de mamoeiro. Agrop Tec. 2015 Jan;36(1)264-272.

19. Araújo EF, Aguiar AS, Arauco AMS, Gonçalves EO, Almeida KNS. Crescimento e qualidade de mudas de paricá produzidas em substratos à base de resíduos orgânicos. Nativa. 2017 Mar;5(1)16-23.

20. Kratka PC, Correia CRMA. Crescimento inicial de aroeira do sertão (Myracrondruon urundeuva Allemão) em diferentes substratos. Rev Arv. 2015 Mai;39(3)551-559, doi: 10.1590/010067622015000300016.

21. Dresh DM, Scalon SPQ, Mussury RM, Kodama FM. Initial growth of Campomanesia adamantium (Cambess.) O. Berg. seedlings on substrates with different composition and water retention capacities. Biosci J. 2016 Jan/Fev;32(1)1-10, doi: 10.14393/BJ-v32n1a2016-26121.

22. David GQ, Marques GP, Peres WM, Matos DL, Rodrigues C. Uso de extratos vegetais no controle de fitopatógenos em sementes de Cedrela fissilis. Cad Agroecol. 2016 Dez;11(2)2236-7934.

23. Moraes-Neto SP, Gonçalves JLM, Takaki M, Gonçalves JC. Crescimento de mudas de algumas espécies arbóreas que ocorrem na mata atlântica, em função do nível de luminosidade. Rev Arv. 2000 Jan;24(1)3545.

24. Carvalho FA, Nascimento MT, Braga JMA. Estrutura e composição florística do estrato arbóreo de um remanescente de mata atlântica submontana no município de Rio Bonito, RJ, Brasil (Mata Rio Vermelho). Rev Arv. 2007 Jul;31(4)717-730, doi: 10.1590/S0100-67622007000400017.

25. Taiz L, Zeiger E. Fisiologia vegetal. Porto Alegre: Artmed; 2013. 918p.

26. Sanquetta CR, Cadori GC, Costa Junior S, Behling A, Corte APD, Ruza MS. Crescimento de área e índice de área foliar de mudas de Eucalyptus unnii Maiden. em diferentes condições de cultivo. Rev. Bioci. 2014 Ago;20(2)82-89.

27. Lima RLS, Severino LS, Ferreira GB, Sofiatti V, Sampaio LR, Beltrão NEM. Casca de mamona associada a quatro fontes de matéria orgânica para a produção de mudas de pinhão-manso. Rev Ceres. 2011 Mar;58(2)232-237, doi: 10.1590/S0034-737X2011000200015.

28. Silva WZ, Brinate SVB, Tomaz MA, Amaral FT, Rodrigues WN, Martins LD. Métodos de estimativa de área foliar em cafeeiro. Enc Biosf. 2011 7(13)746-759.

29. Kitao M, Lei TT, Koike T, Tobita H, Maruyama Y. Susceptibility to photoinhibition of three deciduous broadleaf tree species with different successional traits raised under various light regimes. Plant, Cell \& Environ. 2000 Dez;23(1)81-89, doi: 10.1046/j.1365-3040.2000.00528.x.

30. Marimon BS, Felfili JM, Marimon Júnior BH, Franco AC, Fagg CW. Desenvolvimento inicial e partição de biomassa de Brosimum rubescens Taub. (Moraceae) sob diferentes níveis de sombreamento. Acta Bot Bras. 2008 Out;22(4)941-953, doi: 10.1590/S0102-33062008000400005.

31. Marana JP, Miglioranza E, Fonseca EP. Qualidade de mudas de jaracatiá submetidas a diferentes períodos de sombreamento em viveiro. Rev Arv. Mar;39(2)275-282, doi: 10.1590/0100-67622015000200007.

32. Fonseca EP, Valéri SV, Miglioranza E, Fonseca NAN, Couto L. Padrão de qualidade de mudas de Trema micrantha (L.) Blume, produzidas sob diferentes períodos de sombreamento. Rev Arv. 2002 Jul;26(4)515523, doi: 10.1590/S0100-67622002000400015.

33. Hunt GA. Effect of styroblock design and cooper treatment on morphology of conifer seedlings. In: Rose R, Campbell SJ, Landis TD. Target seedling symposium, meeting of the western forest nursery associations, general technichal report RM-200. 1990, Roseburg: Proceedings... Collins: United States Departament of Agriculture, Forest Service, 1990. p.218-222.

34. Azevedo IMG, Alencar RM, Barbosa AP, Almeida NO. Estudo do crescimento e qualidade de mudas de marupá (Simarouba amara Aubl.) em viveiro. Acta Amaz. 2010 Mar;40(1)157-164, doi: 10.1590/S004459672010000100020.

35. Leles PSS, Lisboa AC, Oliveira Neto SN, Grugiki MA, Ferreira MA. Qualidade de mudas de quatro espécies florestais produzidas em diferentes tubetes. Fl e Amb. 2006 Jan;13(1)69-78.

36. Ajalla ACA, Volpe E, Vieira MC, Zárate NAH. Produção de mudas de baru (Dipteryx alata vog.) sob três níveis de sombreamento e quatro classes texturais de solo. Rev Bras Frut. 2012 Set;34(3)888-896, doi: 10.1590/S0100-29452012000300031. 